\title{
Curcumin attenuates hyperglycemia-driven EGF-induced invasive and migratory abilities of pancreatic cancer via suppression of the ERK and AKT pathways
}

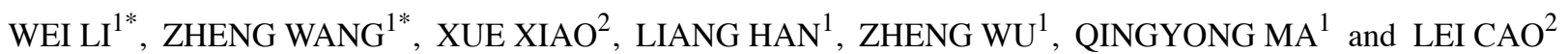 \\ ${ }^{1}$ Department of Hepatobiliary Surgery, The First Affiliated Hospital of Xi'an Jiaotong University; \\ ${ }^{2}$ Department of Pharmacology, School of Basic Medical Sciences, Xi'an Jiaotong University \\ Health Science Center, Xi'an, Shaanxi 710061, P.R. China
}

Received June 8, 2018; Accepted October 12, 2018

DOI: 10.3892/or.2018.6833

\begin{abstract}
The relationship between diabetes mellitus and pancreatic cancer is complex. Diabetes has been postulated to be both an independent risk factor and a consequence of pancreatic cancer. Our previous study confirmed that curcumin plays a vital role in inhibiting the epithelial-mesenchymal transition of pancreatic cancer cells. However, whether curcumin attenuates hyperglycemia-induced cancer invasive and migratory abilities and the underlying mechanisms are not yet well understood. As high glucose is able to induce the expression of epidermal growth factor (EGF), which is intimately related with tumor progression, the aim of this study was to evaluate whether curcumin could influence the high glucose-induced EGF/EGFR pathway and the biological activity of pancreatic cancer cells. Human pancreatic cancer BxPC-3 cells were exposed to high glucose or EGF, with or without curcumin, LY 294002 (an Akt inhibitor) or PD 98059 (an ERK inhibitor). MTT, Transwell invasion and wound healing assays were used to detect the proliferation, invasion and migration potential of cancer cells. The activation of p-EGFR, p-ERK and p-Akt was determined by western blot analysis. The expression levels of UPA and E-cadherin were examined using qRT-PCR and western blot analysis. The results showed that high glucose could not only promote the proliferation, invasion and migration of pancreatic cancer cells, but also induce the expression of EGF and activation of EGFR, ERK and Akt. These effects of high glucose were counter-balanced by curcumin.
\end{abstract}

Correspondence to: Dr Lei Cao, Department of Pharmacology, School of Basic Medical Sciences, Xi'an Jiaotong University Health Science Center, 76 West Yanta Road, Xi'an, Shaanxi 710061, P.R. China

E-mail: 289421999@qq.com; leicao@mail.xjtu.edu.cn

*Contributed equally

Key words: curcumin, hyperglycemia, EGF, ERK, Akt, migration, invasion, pancreatic cancer
EGF-induced proliferative, invasive and migratory abilities of BxPC-3 cells were abrogated by curcumin, LY 294002 and PD 98059. In addition, EGF-modulated activation of EGFR, ERK and Akt, as well as the expression of uPA and E-cadherin were inhibited by curcumin. Taken together, the present study indicates that curcumin suppresses hyperglycemia-driven EGF-induced invasion and migration of pancreatic cancer cells by inhibiting the EGF/EGFR signaling pathway and its downstream signaling molecules including ERK and Akt. Curcumin is a potential anticancer agent for pancreatic cancer.

\section{Introduction}

Pancreatic cancer is a highly aggressive and fatal malignant digestive tumor for which the 5-year relative survival rate is $8 \%$. The poor prognosis of this severe disease is mainly due to the local invasion and distant metastasis at an early stage. In 2018, 55,440 individuals were estimated to be newly diagnosed with pancreatic cancer, which may result in more than 44,000 cancer-related deaths in the United States (1). In China, pancreatic cancer accounts for the second leading upward trend of age-standardized mortality rates from 2000 to 2011 (2). Routine treatments for this severe disease include surgery, radiation and chemotherapy. However, at present, approximately $80 \%$ of pancreatic cancer patients who are diagnosed do not qualify for surgical resection due to early relapse or metastatic spread of the disease. Therefore, the exploration of risk factors and novel effective therapeutic options are urgently needed to improve the treatment outcome in patients with pancreatic cancer (3).

Diabetes mellitus could be both a risk factor and a consequence of pancreatic cancer. A collaborative analysis from the International Pancreatic Cancer Case-Control Consortium analyzed more than 8,000 pancreatic cancer cases and confirmed that individuals with diabetes mellitus have an almost 2-fold increased risk of developing pancreatic cancer (4). In a Chinese retrospective cohort study, both male and female type 2 diabetes mellitus patients had an increased risk to develop pancreatic cancer compared with the general population with the standardized incidence ratios of 2.973 and 2.687, respectively (5). Diabetes mellitus improves following 
pancreatectomy, suggesting that diabetes may be induced by pancreatic cancer (6). Our previous studies confirmed that a hyperglycemic condition could promote the proliferation, invasion, epithelial-mesenchymal transition (EMT) as well as the metastasis of pancreatic cancer $(7,8)$.

Epidermal growth factor (EGF) is a polypeptide that regulates various cellular functions involving proliferation, survival, differentiation, angiogenesis and metastasis in many types of cancer. EGF binds with a high affinity receptor located in the cellular membrane and stimulates rapid activation of protein kinase activity (9). Overexpression of epidermal growth factor receptor (EGFR) and ligands has been shown in pancreatic ductal adenocarcinoma and pancreatic cancer cells, which is essential for the initiation and progression of this disease (10). In our previous study, it was shown that high glucose could promote pancreatic cancer cell proliferation via the induction of EGF expression and transactivation of EGFR (11).

Curcumin, a natural polyphenol compound derived from turmeric, has multiple biologic properties, including health maintenance and cancer prevention (12). Curcumin exhibits its antitumor effects via targeting multiple signaling pathways, including mitogen-activated protein kinase (MAPK), protein kinase B (Akt) and others (13). In a recent study, it was indicated that curcumin plays an important role in suppressing superoxide dismutase-induced EMT of pancreatic cancer by inhibiting the PI-3K/Akt/NF- $\mathrm{B}$ signaling pathway (14). It was also confirmed that curcumin could restrain hypoxiainduced EMT via suppression of the hedgehog signaling pathway in pancreatic cancer cells (15). However, whether curcumin is able to inhibit high glucose-induced progression of pancreatic cancer and the related mechanisms have not yet been elucidated.

In the present study, the hypothesis that curcumin is able to inhibit high glucose-driven EGF-induced invasive and migratory abilities in pancreatic cancer cells was tested. The effect of curcumin on high glucose-induced activation of EGFR, extracellular signal-regulated kinase (ERK) and Akt signaling pathways was also investigated. In addition, the effect of curcumin on EGF-modulated activation of EGFR, ERK and Akt, as well as the expression of uPA and E-cadherin was demonstrated. Results from this study suggest that curcumin is a potential anticancer agent for the therapy of pancreatic cancer via the suppression of the EGF/EGFR signaling pathway and its downstream signaling molecules including ERK and Akt.

\section{Materials and methods}

Cell culture and reagents. Human BxPC-3 pancreatic cancer cell line was obtained from the American Type Culture Collection (ATCC; Manassas, VA, USA). The cells were cultured in Gibco ${ }^{\mathrm{TM}}$ DMEM (Thermo Fisher Scientific, Inc., Waltham, MA, USA), which contained $10 \%$ dialyzed heatinactivated FBS, $100 \mathrm{U} / \mathrm{ml}$ penicillin as well as $100 \mu \mathrm{g} / \mathrm{ml}$ streptomycin in a humidified atmosphere of $5 \% \mathrm{CO}_{2}$ at $37^{\circ} \mathrm{C}$. Exponentially growing cells in complete medium were treated with $50 \mathrm{ng} / \mathrm{ml}$ EGF, $20 \mu \mathrm{M}$ curcumin, $10 \mu \mathrm{M}$ LY 294002 (a PI3K inhibitor), and/or 50 $\mu \mathrm{MPD} 98059$ (an ERK inhibitor) in normal culturing conditions (5.5 mM glucose) or high glucose ( $25 \mathrm{mM}$ ) conditions for indicated time intervals according to the aim of the experiment. Curcumin, EGF, PD 98059 and
LY294002 were purchased from Sigma-Aldrich; Merck KGaA (Darmstadt, Germany). Millicell ${ }^{\circledR}$ culture plate inserts for the Transwell assay were obtained from EMD Millipore (Billerica, MA, USA). Matrigel was purchased from BD Biosciences (Bedford, MA, USA). Primary antibodies [dilution at 1:100 in phosphate-buffered saline (PBS)-Tween-20] against EGF (cat. no. sc-374255), E-cadherin (cat. no. sc-52328) and uPA (cat. no. sc-59727) were obtained from Santa Cruz Biotechnology (Santa Cruz, CA, USA). The anti-EGFR (cat. no. 4267), anti-phospho-EGFR (Tyr 1068, cat. no. 2234), anti-Akt (cat. no. 9272), anti-phospho-Akt (Ser473, cat. no. 4060), anti-ERK (cat. no. 9102) and anti-phospho-ERK (Thr202/Tyr204, cat. no. 9106) were purchased from Cell Signaling Technology (Beverly, MA, USA). Other reagents were purchased from common commercial sources. All drug solutions were freshly prepared on the day of testing.

MTT proliferation assays. BxPC-3 cells were seeded in 96-well plates at the density of $1 \times 10^{4}$ cells per well. The cells were treated with curcumin, PD 98059 or LY 294002 in EGF or a high glucose condition. After incubation for $24 \mathrm{~h}$ at $37^{\circ} \mathrm{C}$, $15 \mu \mathrm{l}$ of MTT solution was added to each well and the cells were incubated for $4 \mathrm{~h}$ at $37^{\circ} \mathrm{C}$. DMSO $(100 \mu \mathrm{l})$ was then added to each well. The optical density (OD) value at $490 \mathrm{~nm}$ was determined using a spectrophotometer (Bio-Rad Laboratories, Inc., Hercules, CA, USA).

Transwell Matrigel invasion assay. The $8.0-\mu \mathrm{m}$ pore inserts of the Transwell chambers were coated with $30 \mu \mathrm{l}$ of Matrigel. After serum starvation for $24 \mathrm{~h}, \mathrm{BxPC}-3$ cells were suspended in the top chamber at a concentration of $5 \times 10^{4}$ in DMEM containing $1 \% \mathrm{FBS}$ in the absence or presence of high glucose, EGF, curcumin, PD 98059 and/or LY 294002. Simultaneously, in the lower chambers, $500 \mathrm{ml}$ of DMEM containing 20\% FBS was placed. The Matrigel invasion chamber was incubated for $48 \mathrm{~h}$ in a humidified tissue culture incubator. The non-invading cells were then removed from the upper surface. After staining with crystal violet, the stained cells on the bottom surface were counted under an Nikon inverted microscope DIAPHOT-TMD (Nikon, Tokyo, Japan) to test the invasion ability of the cancer cells. Three random fields were captured at x20 magnification $(n=3)$.

Wound healing assay. BxPC-3 cells $\left(1.0 \times 10^{5}\right.$ cells $\left./ 500 \mu \mathrm{l}\right)$ were seeded in 24-well plates. A sterile pipette tip was used to produce a wound line between the cells that grew to 90-100\% confluence and cellular debris was removed. Cells were allowed to migrate for $24 \mathrm{~h}$. Images were captured at time 0 and 24 h post-wounding under a Nikon Diaphot TMD inverted microscope (x10 magnification). The relative distance traveled by the leading edge from 0 to $24 \mathrm{~h}$ was assessed using Photoshop software (Adobe Systems, Inc., San Jose, CA, USA) $(n=5)$.

Real-time quantitative PCR ( $q R T-P C R)$. After being extracted from the BxPC-3 cells using the Fastgen 200 RNA isolation system (Fastgen, Shanghai, China) according to the manufacturer's protocol, the total RNA was reverse-transcribed into cDNA using the Fermentas RevertAid ${ }^{\mathrm{TM}}$ kit (MBI Fermentas, Burlington, ON, Canada). The primer sequences 
were as follows: uPA-F,5'-TAA GAG CTG GTG TCT GAT TG-3' and uPA-R, 5'-TTG GAT GAA CTA GGC TAA AA-3'; E-cadherin-F, 5'-ATTCTGA TTCTGCTGCTCTTG-3' and E-cadherin-R, 5'-AGTCCTGGTCCTCTTCTCC-3'; $\beta$-actin-F, 5'-GACTTAGTTGCGTTACACCCTTTCT-3' and $\beta$-actin-R, 5'-GAACGGT GAAGGTGACAGCAGT-3'. The PCR reactions consisted of $30 \mathrm{sec}$ at $95^{\circ} \mathrm{C}$, followed by 40 cycles of $95^{\circ} \mathrm{C}$ for $5 \mathrm{sec}, 60^{\circ} \mathrm{C}$ for $30 \mathrm{sec}$ and $72^{\circ} \mathrm{C}$ for $30 \mathrm{sec}$. After each qRT-PCR experiment, a dissociation curve analysis was conducted. The relative gene expression was calculated using the previously described $2^{-\Delta \Delta C q}$ method (16).

Western blot analysis. Total protein from BxPC-3 cells was extracted from cultured cells in Radio-immunorrecipitation assay (RIPA) lysis buffer on ice for $25 \mathrm{~min}$. Insoluble materials were removed by centrifugation at $4^{\circ} \mathrm{C}$ with $15,000 \mathrm{x}$ g for 15 min. Subsequently, supernatants were collected and total protein concentrations were measured using the BCA assay kit (Pierce, Rockford, IL, USA). Clarified protein lysates (30-80 $\mu \mathrm{l}$ ) from the BxPC-3 cancer cells were electrophoretically resolved on a denaturing SDS-polyacrylamide gel (10-12\%) and electrotransferred onto polyvinylidene difluoride (PVDF) membranes. The membranes were initially blocked with 5\% non-fat dry milk in Tris-buffered saline (TBS) for $2 \mathrm{~h}$ and then probed with antibodies against E-cadherin, uPA, EGF, EGFR, p-EGFR, Akt, p-Akt, ERK, p-ERK and $\beta$-actin (Control). After incubation with the primary antibodies at $4^{\circ} \mathrm{C}$ overnight, the membranes were hybridized with secondary goat anti-mouse (cat. no. 7056) or goat anti-rabbit (cat. no. 7074) antibodies at 1:1,000 dilution (Cell Signaling Technology, Beverly, MA, USA) for $2 \mathrm{~h}$ at room temperature. Immunopositive bands were developed using an enhanced chemiluminescence (ECL) detection system (Amersham, Piscataway, NJ, USA). Immunodetection was visualized on a Gel Doc 2000 Imaging System (Bio-Rad Laboratories). All analyses were conducted in triplicate. The data were analyzed as (p-protein/control)/(total protein/control).

Statistical analysis. Statistical analysis was performed using SPSS software (version 17.0; SPSS Inc., Chicago, IL, USA). Data are presented as the means \pm SEM of three replicate assays. Differences between the groups were analyzed by analysis of variance [ANOVA followed by least significant difference (LSD)]. Statistical significance was set at $\mathrm{P}<0.05$. All experiments were repeated independently at least three times.

\section{Results}

Curcumin inhibits high glucose and EGF-induced proliferation in pancreatic cancer cells. Our previous study demonstrated that a hyperglycemic condition promotes the proliferation of pancreatic cancer cells via the induction of EGF expression as well as the transactivation of EGFR (11). As our previous studies found that curcumin shows a $50 \%$ inhibitory concentration $\left(\mathrm{IC}_{50}\right)$ of $\sim 20 \mu \mathrm{M}$ and this concentration exhibited no cytotoxic effects in BxPC-3 cells, the treatment concentration of $20 \mu \mathrm{M}$ of curcumin was used in the present experiments (17). Here, it was shown that both a high glucose condition and EGF stimulation accelerated the growth

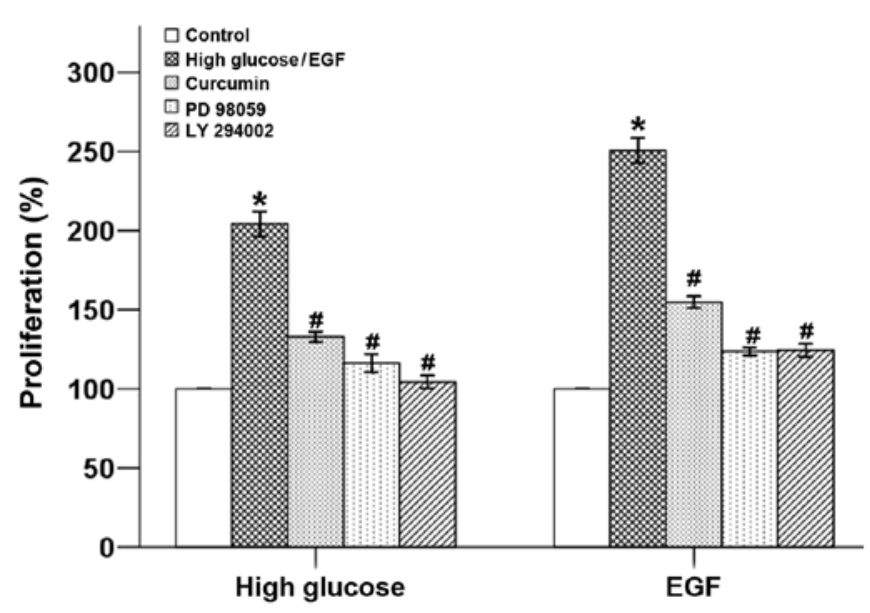

Figure 1. Effects of curcumin on high glucose- or EGF-induced pancreatic cancer cell proliferation. BxPC-3 cells were treated with normal glucose (control, $5.5 \mathrm{mM})$, high glucose $(25 \mathrm{mM})$ or EGF $(50 \mathrm{ng} / \mathrm{ml})$ condition with or without $20 \mu \mathrm{M}$ curcumin, $10 \mu \mathrm{M}$ LY 294002 (a PI3K inhibitor), $50 \mu \mathrm{M}$ PD 98059 (an ERK inhibitor) for $24 \mathrm{~h}$ to analyze the cell proliferation rates. ${ }^{*} \mathrm{P}<0.05$ as compared with the control group (normal glucose, $5.5 \mathrm{mM}$ ); ${ }^{\#} \mathrm{P}<0.05$ as compared with the high glucose group (25 mM) or EGF group. EGF, epidermal growth factor.

of BxPC-3 cells, which was able to be counter -balanced by curcumin. In addition both PD 98059 and LY 294002 inhibited the high glucose- and EGF-induced cellular proliferation, which indicated that the growth of tumor cells was mediated via the ERK and Akt pathways (Fig. 1).

Curcumin inhibits high glucose-induced invasive ability and wound closure of pancreatic cancer cells. Invasion and migration of cancer cells are considered detrimental aspects in the development of tumor metastasis. In order to confirm whether curcumin influences high glucose-induced pancreatic cancer cell invasion and migration, Transwell invasion and woundhealing assays were conducted. As shown in Fig. 2, the average cell numbers that invaded into the lower chamber were significantly increased in the high glucose condition after incubation for $48 \mathrm{~h}$ and this increase was reversed by co-treatment with curcumin. The migratory ability of BxPC-3 cells after incubation for $24 \mathrm{~h}$ was also suppressed by curcumin.

Curcumin downregulates high glucose-induced activation of EGF/ERK and EGF/Akt pathways. In order to form metastases, cancer cells undergo various steps, including local invasion, intravasation, survival in the circulation, arrest at distant organ site, micrometastasis formation and metastatic colonization (18). Different molecular pathways are activated to pass these important steps, such as the ERK pathway and PI-3K/Akt pathway (18). The ERK pathway, which belongs to the mitogen-activated protein kinase (MAPK) signaling pathway, is involved in tumor proliferation, differentiation, migration and invasion (19). The PI3K/Akt pathway promotes cancer development, angiogenesis as well as metastasis (20).

Our previous study confirmed that high glucose could promote the expression of EGF and transactivation of EGFR (11). Hyperglycemic conditions could also activate the ERK and p38 MAPK signaling pathways via the production of $\mathrm{H}_{2} \mathrm{O}_{2}$ in 

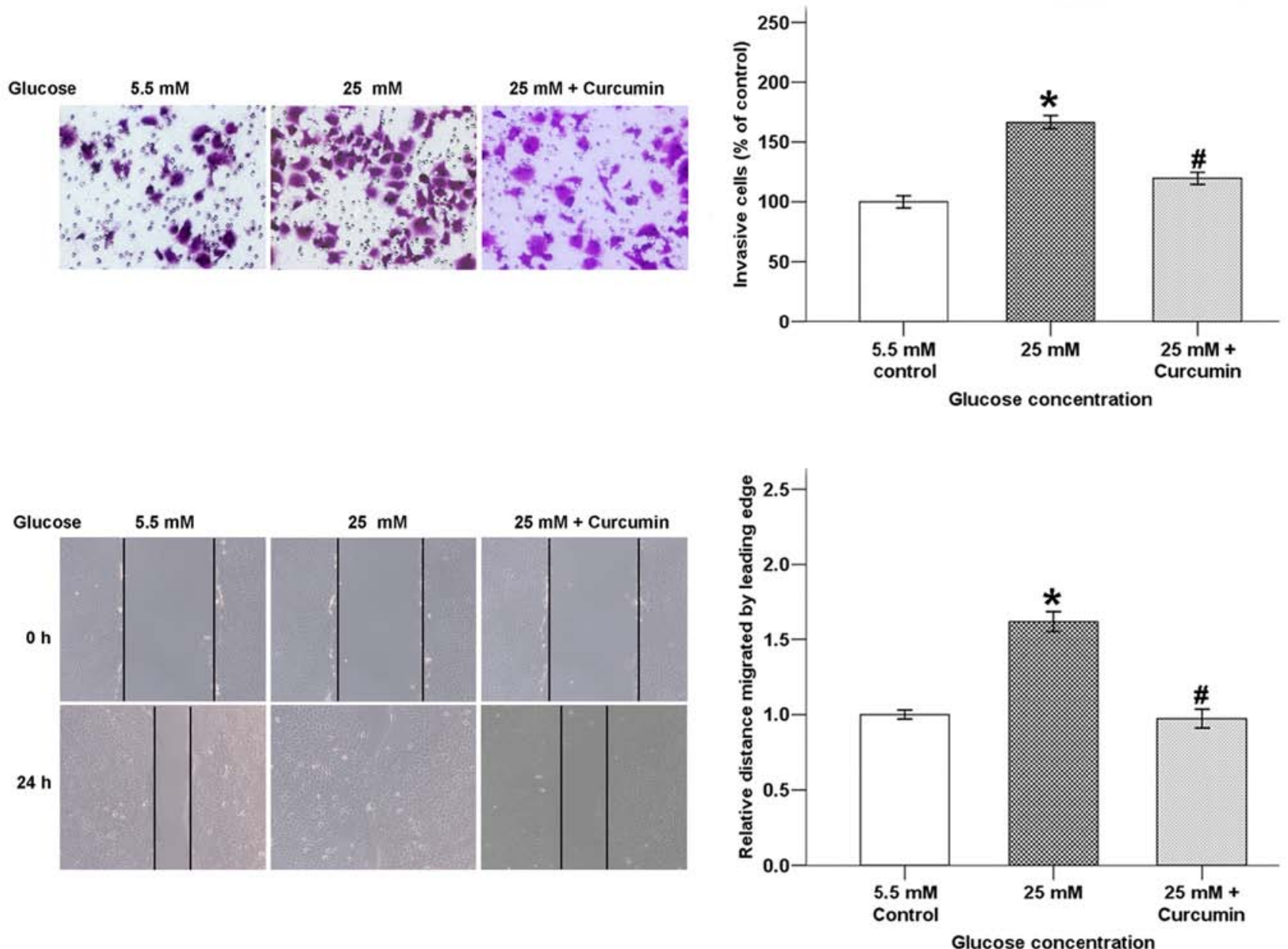

Figure 2. Effects of curcumin on the high glucose-induced invasive and migratory ability of cancer cells. Pancreatic cancer cells were exposed to normal glucose $(5.5 \mathrm{mM})$, high glucose $(25 \mathrm{mM})$ condition as well as $20 \mu \mathrm{M}$ curcumin for 24 or $48 \mathrm{~h}$. The number of migrated cells was quantified by counting the number of cells at $\times 200$ magnification after $48 \mathrm{~h}$ treatment. The confluent monolayer was wounded with a sterile pipette tip and the cells were allowed to migrate for $24 \mathrm{~h} .{ }^{*} \mathrm{P}<0.05$ as compared with the $5.5 \mathrm{mM}$ glucose group; ${ }^{\prime \prime} \mathrm{P}<0.05$ as compared with the $25 \mathrm{mM}$ glucose group.

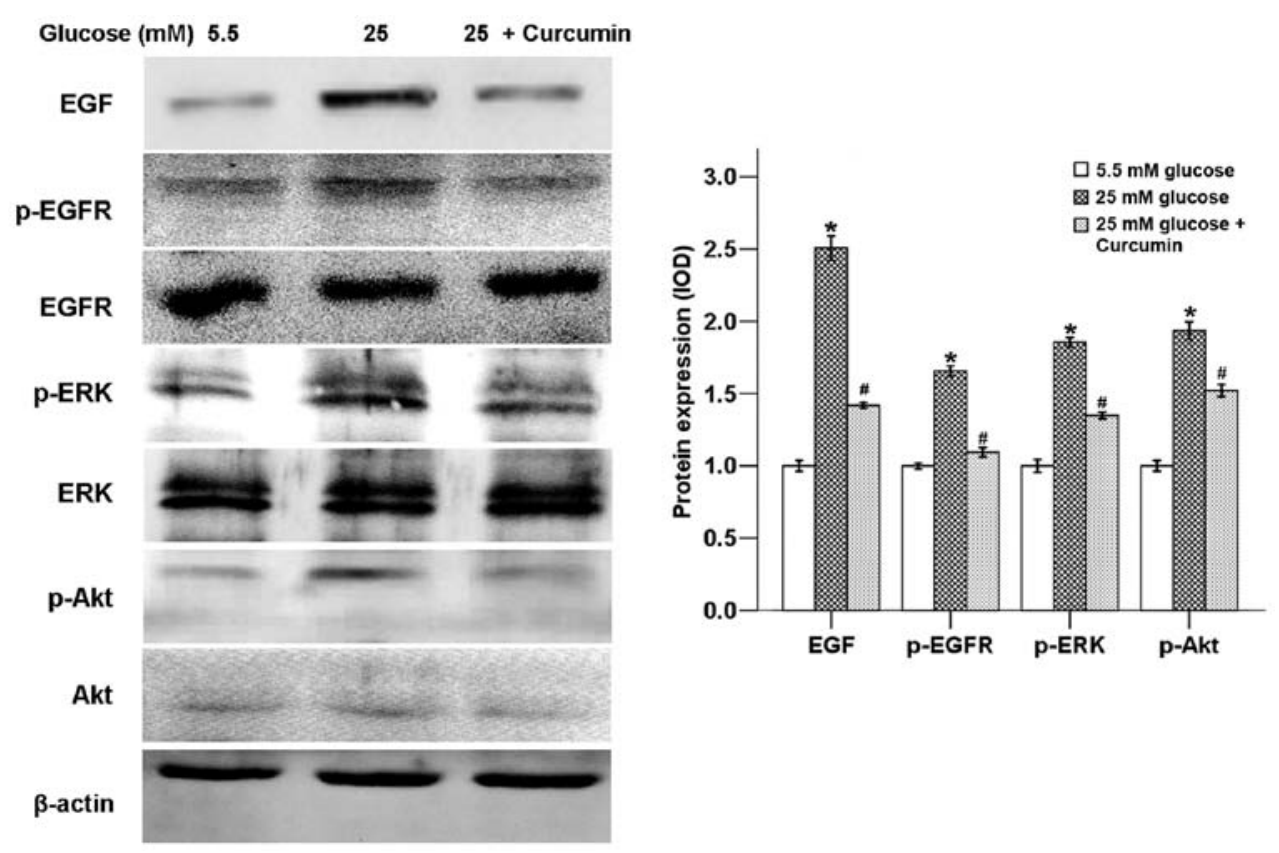

Figure 3. Role of curcumin in high glucose-induced EGF expression and phosphorylation of EGFR, ERKas well asAkt. BxPC-3 cells were treated with $20 \mu \mathrm{M}$ curcumin for $24 \mathrm{~h}$ under high glucose $(25 \mathrm{mM})$ or normal glucose $(5.5 \mathrm{mM})$ condition to evaluate the expression of EGF and the phosphorylation levels of EGFR, ERK and Akt. "P $<0.05$ as compared with the $5.5 \mathrm{mM}$ glucose group; ${ }^{*} \mathrm{P}<0.05$ as compared with the $25 \mathrm{mM}$ glucose group. EGF, epidermal growth factor; EGFR, epidermal growth factor receptor; ERK, extracellular signal-regulated kinase; Akt, protein kinase B. 

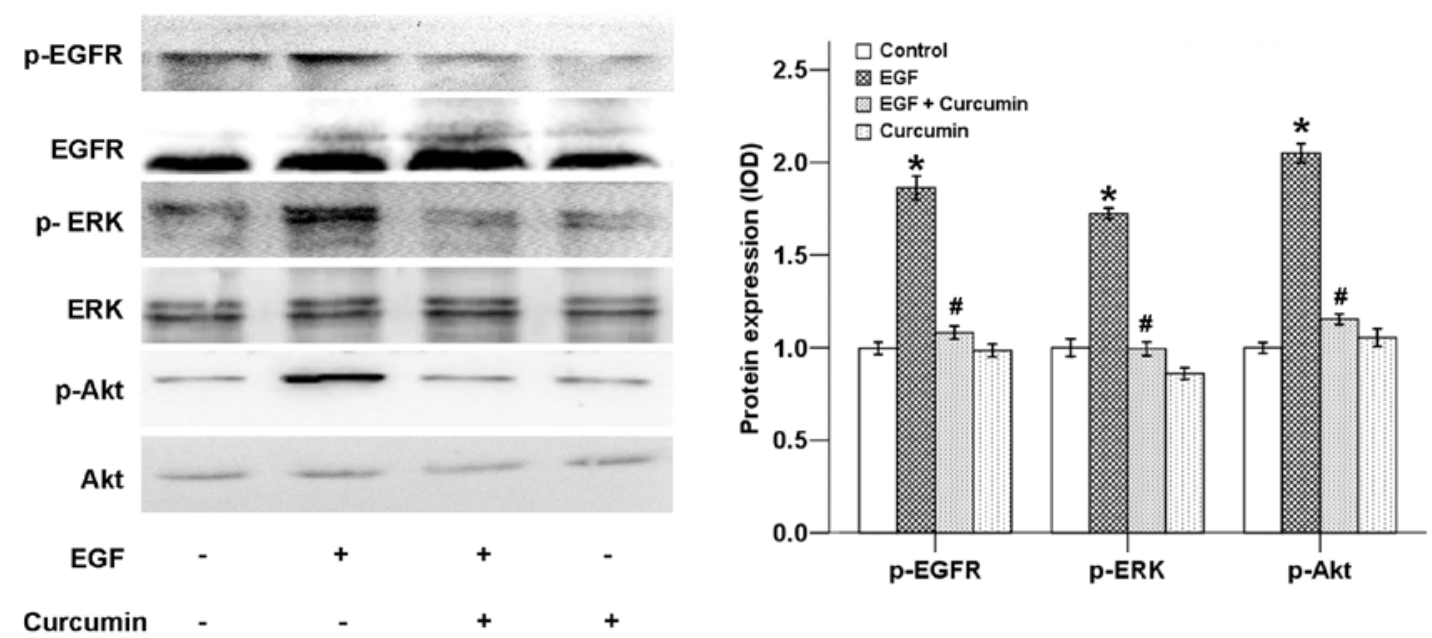

Figure 4. Role of curcumin in EGF-induced phosphorylation of EGFR, ERK and Akt. BxPC-3 cells were treated with $20 \mu \mathrm{M}$ curcumin for $24 \mathrm{~h}$ with or without EGF $(50 \mathrm{ng} / \mathrm{ml})$ to evaluate the phosphorylation levels of EGFR, ERK and Akt. ${ }^{*} \mathrm{P}<0.05$ as compared with the control group; ${ }^{*} \mathrm{P}<0.05$ as compared with the EGF group. EGF, epidermal growth factor; EGFR, epidermal growth factor receptor; ERK, extracellular signal-regulated kinase; Akt, protein kinase B.

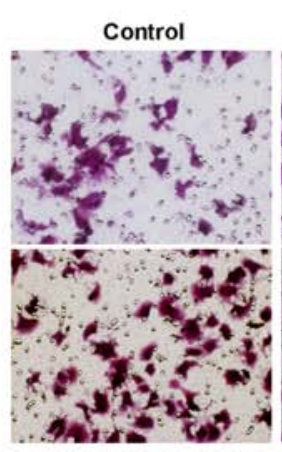

EGF + Curcumin

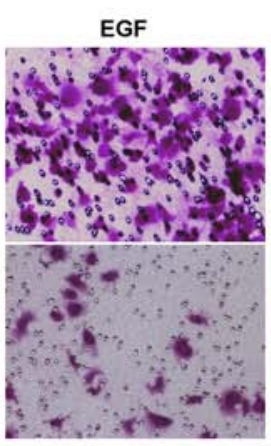

EGF + PD 98059



EGF + LY 294002

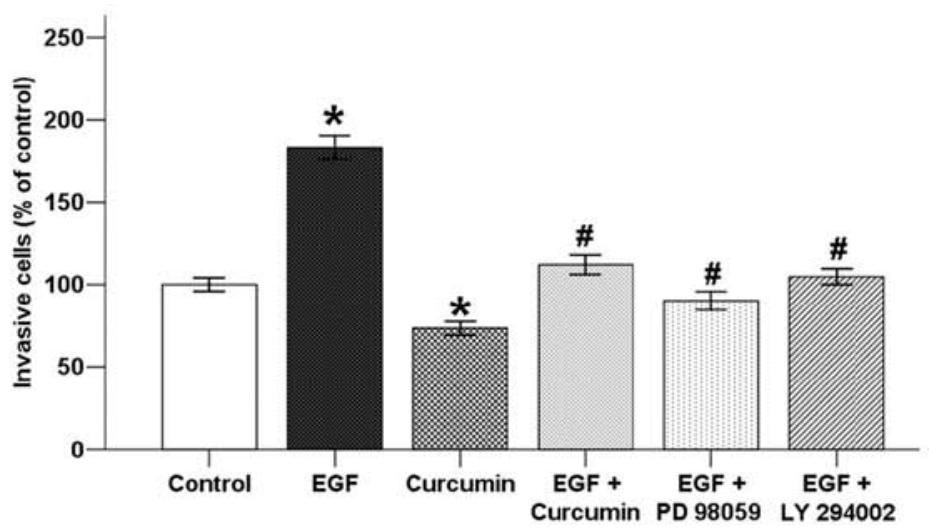

Figure 5. Effects of curcumin on EGF-induced invasive ability of pancreatic cancer cells. Pancreatic cancer cells were exposed to EGF (50 ng/ml) as well as $20 \mu \mathrm{M}$ curcumin, $50 \mu \mathrm{M}$ PD 98059 (an ERK inhibitor) or $10 \mu \mathrm{M}$ LY 294002 (a PI3K inhibitor) for $48 \mathrm{~h}$, and the number of migrated cells was quantified by counting the number of cells at $\mathrm{x} 200$ magnification. ${ }^{*} \mathrm{P}<0.05$ as compared with the control group; ${ }^{\#} \mathrm{P}<0.05$ as compared with the EGF group. EGF, epidermal growth factor.

pancreatic cancer (21). In this study, it was shown that a high glucose condition activated ERK and Akt signaling pathways, as both ERK and Akt phosphorylation were strongly increased. Curcumin was able to suppress the expression of EGF and activation of EGFR, ERK and Akt in the high glucose condition (Fig. 3).

In order to assess whether the high glucose-induced activation of ERK and Akt signaling pathways was EGF/ EGFR-dependent, BxPC-3 cells were treated with EGF. As shown in Fig. 4, EGF significantly promoted the phosphorylation of EGFR, ERK and Akt, while curcumin was able to counter-balance these effects of EGF.

Curcumin inhibits EGF-induced invasive ability of pancreatic cancer cells. Growth factors, such as EGF and vascular endothelial growth factor (VEGF), are intimately related with cancer cell migration, angiogenesis, regulation of cell adhesion and EMT (22). Activation of ErbB ligands and overexpression of EGFR significantly promote tumor metastasis via chemotaxis and intravasation (23).
In order to confirm whether curcumin inhibits EGF-induced BxPC-3 cell invasion, a Transwell invasion assay was utilized. As shown in Fig. 5, the number of cells that invaded into the lower chamber was increased with the addition of EGF after incubation for $48 \mathrm{~h}$. This increase was reversed by co-treatment with curcumin. In addition, both PD 98059 and LY 294002 also suppressed the effect of EGF which indicated that EGF-induced invasion was related to the ERK and Akt pathways.

Curcumin suppresses EGF-induced wound closure of pancreatic cancer cells. A classic wound healing assay was used to evaluate the effect of curcumin on EGF-induced BxPC-3 cell motility. The results showed that EGF significantly increased the migratory ability of the BxPC-3 cells after incubation for $24 \mathrm{~h}$. Curcumin counter-balanced these effects of EGF. Both PD 98059 and LY 294002 inhibited EGF-induced wound closure of pancreatic cancer cells. Curcumin exerts its inhibitory effect on cellular motility, which might be attributed to the suppression of the EGF/ERK and EGF/Akt pathways (Fig. 6). 

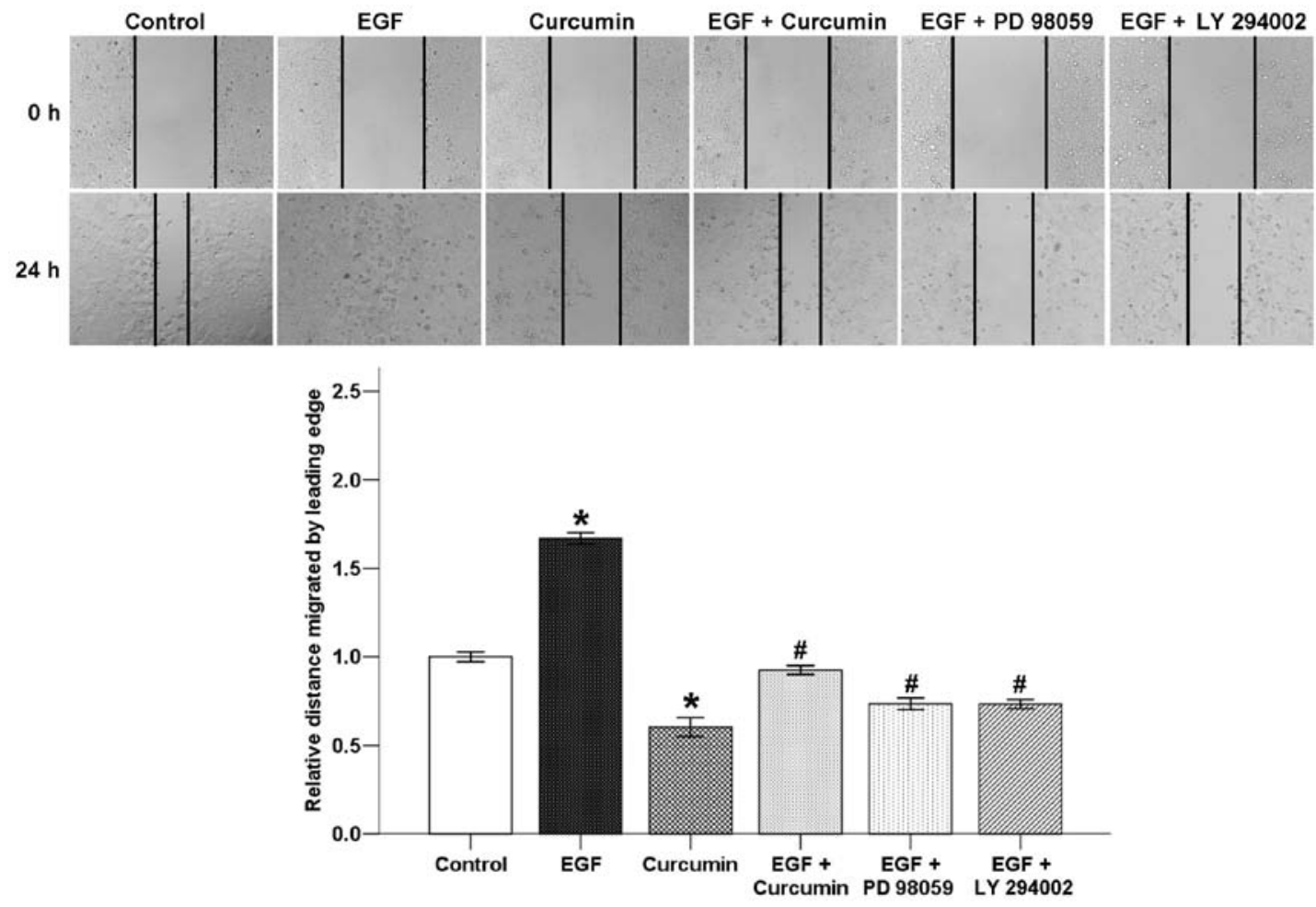

Figure 6. Effects of curcumin on EGF-induced migration of BxPC-3 cells.The confluent monolayer was wounded with a sterile pipette tip, and the cancer cells were allowed to migrate for $24 \mathrm{~h}$. The migratory ability of BxPC-3 cells was enhanced by EGF, whereas the addition of $20 \mu \mathrm{M}$ curcumin, $50 \mu \mathrm{M}$ PD 98059 (an ERK inhibitor) or $10 \mu \mathrm{M} \mathrm{LY} 294002$ (a PI3K inhibitor) reduced the migration of pancreatic cancer cells. "P<0.05 as compared with the control group; ${ }^{\text {"}} \mathrm{P}<0.05$ as compared with the EGF group. EGF, epidermal growth factor.

Curcumin inhibits EGF-modulated expression of metastaticrelated factors. Our previous study demonstrated that a high glucose condition could induce the invasive and migratory abilities of pancreatic cancer cells by regulating metastaticrelated factors, including uPA and E-cadherin (24). In the present study, it was shown that EGF direct stimulation also promoted the mRNA expression of UPA and down-regulated the mRNA level of E-cadherin. Curcumin significantly reversed these EGF-induced effects (Fig. 7). In addition, curcumin also reversed the EGF-modulated metastatic-related factors at the protein level, and the trend was consistent with the mRNA results. Taken together, these results indicate that curcumin inhibits invasion and migration of pancreatic cancer cells under high glucose conditions, which maybe attributed to the EGF/ERK and EGF/Akt signaling pathways.

\section{Discussion}

Due to local recurrence, lymphnode and liver metastases as well as peritoneal dissemination, pancreatic cancer is one of the most aggressive malignant diseases, the hallmarks of which includes poor outcome, short survival duration and resistance to therapy (25). In China, pancreatic cancer is the seventh deadliest disease with annual mortality rates almost equal to incidence rates, of which the 5-year relative survival rate is $4.1 \%$ and the median survival time is 3.9 months (6). The exploration of risk factors and newer effective therapeutic options are important to improve the treatment outcome for pancreatic cancer patients. Diabetes mellitus can be both a risk factor and a consequence of pancreatic cancer. Our previous study confirmed that high glucose could promote pancreatic cancer cell proliferation via the induction of epidermal growth factor (EGF) expression and transactivation of the epidermal growth factor receptor (EGFR) (11). Hyperglycemic conditions could worsen the prognosis of pancreatic cancer by enhancing invasive ability and promoting epithelial-mesenchymal transition (EMT) through the production of hydrogen peroxide (8). In addition, diabetes mellitus was also found to enhance perineural invasion in pancreatic cancer patients and to aggravate a poor prognosis (7). It has been verified that the addition of 5.5 and $25 \mathrm{mM}$ glucose resulted in balanced osmotic pressure inside and outside the cell membrane (21), thus we used 5.5 and $25 \mathrm{mM}$ of glucose as it has been applied worldwide in the related field as a classical high glucose model in the cell culture. In the present study, we focused on whether curcumin is able to suppress high glucose-induced cancer proliferation, migratory and invasive abilities and the underlying mechanism.

Our data showed that a high glucose condition could promote the proliferation, migration and invasion of pancreatic cancer cells. High glucose was not only able to increase the expression of EGF and transactivation of EGFR, but also activate the ERK and Akt signaling pathways. Curcumin was able to abrogate these effects of a high glucose condition. In addition, in order to ascertain whether the effect of a high glucose condition on pancreatic cancer cells was EGF-dependent, we also treated BxPC-3 cells with EGF directly. Data showed that EGF treatment significantly promote the proliferative, migratory and invasive abilities of the BxPC-3 cells. EGF treatment also modulated the expression levels of p-EGFR, 

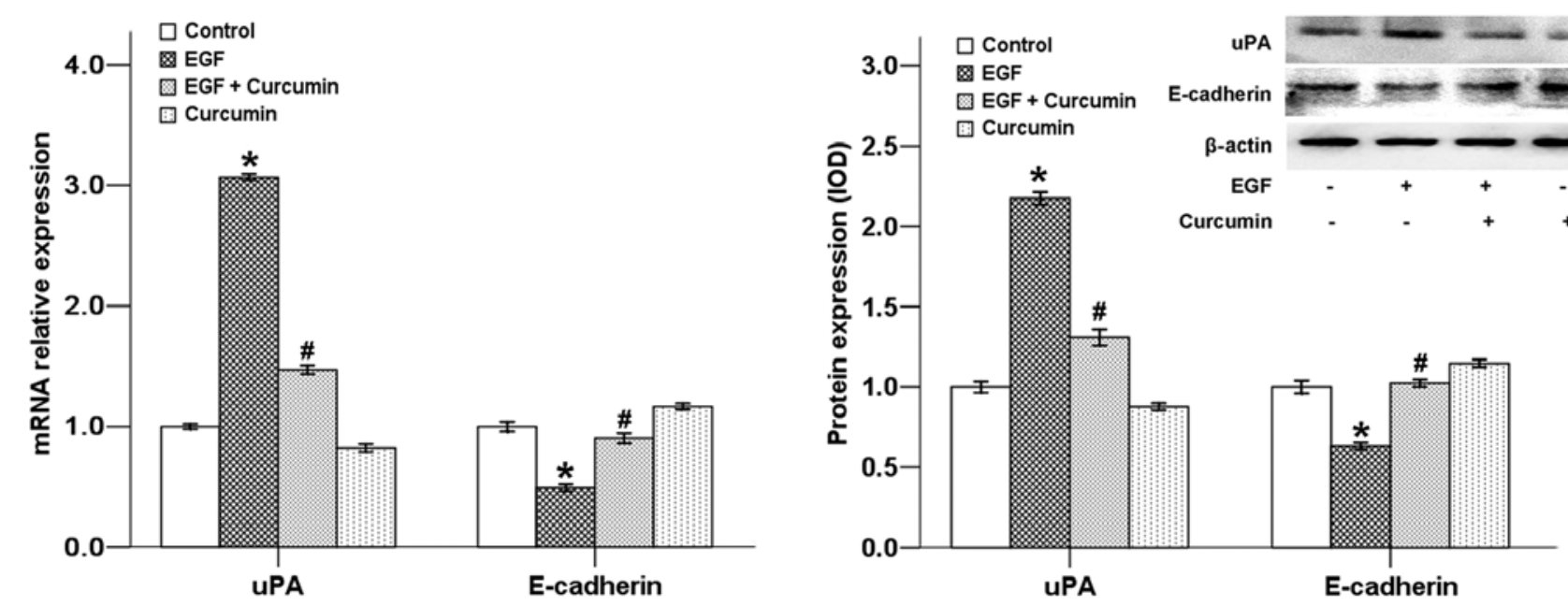

Figure 7. Effects of curcumin on the high glucose-modulated expression of uPA and E-cadherin. BxPC-3 cells were treated with $20 \mu \mathrm{M}$ curcumin and/or $50 \mathrm{ng} / \mathrm{ml} \mathrm{EGF}$ and the expression of uPA and E-cadherin at the mRNA and protein levels was determined. ${ }^{*} \mathrm{P}<0.05$ as compared with the control group; ${ }^{*} \mathrm{P}<0.05$ as compared with the EGF group. EGF, epidermal growth factor.

p-ERK, p-Akt, uPA and E-cadherinin pancreatic cancer cells. Curcumin was also able to suppress the effects of EGF treatment. The addition of PD 98059 and LY 294002 to the cell culture resulted in the inhibition of cellular growth and invasion. Our results indicate that curcumin inhibits high glucose-induced invasion and migration of pancreatic cancer cells, which might be attributed to the EGF/ERK and EGF/Akt signaling pathways.

EGF belongs to the EGF family, which includes EGF, transforming growth factor $\alpha($ TGFA), amphiregulin, betacellulin,cripto, heparin-binding EGF (HB-EGF), epigen, epiregulin and neuregulin.EGF exertsits biological effects by binding to the receptors in an autocrine or paracrine manner. The ErbB family consists of four receptors: EGFR (ErbB-1), ErbB-2, ErbB-3 and ErbB-4. After binding of EGF, the four members of the family are able to form various heterodimers, which lead to autophosphorylation and further activatedownstream signaling pathways, including the PI3K/Akt pathway and MAPKs, such as the ERK pathway (9). The signaling cascades activated by EGF/EGFR could further regulate different cellular responses such as proliferation, survival, migration, invasion, intravasation and metastasis (26).

The ERK MAPK signaling pathway is involved in the regulation of several cancer cell processes, including proliferation, differentiation, survival, cellular motility and metastasis. Aberrant signaling drives tumor initiation and progression. The PI3K/Akt/mTOR signaling pathway also promotes cancer development, migration, invasion, angiogenesis and metastasis (18). Overexpression or mutational activation of EGFR has been found in cancer cells leading to activation of the ERK and PI3K/Akt signaling pathways (27). Various EGFR inhibitors have been developed for cancer treatment, including EGFR monoclonal antibodies (cetuximab, panitumumab) and tyrosine kinase inhibitors, including erlotinib, gefitinib and lapatinib.These drugs have been successfully approved for the treatment of metastatic colorectal cancer (cetuximab and panitumumab), locally advanced, unresectable, or metastatic pancreatic cancer (erlotinib), breast cancer (lapatinib) and non-small cell lung cancer (gefitinib and erlotinib) (28). In the present study, it was found that high glucose and EGF stimulation could activate EGFR, ERK and Akt, which further induce cancer proliferation, migration and invasion. As a type of natural polyphenol compound derived from turmeric,curcumin was able to abrogate these effects leading to cancer prevention.

Accumulating evidence suggests that curcumin inhibits cancer initiation promotion and progression through regulation of multiple signaling pathways including EGF/EGFR, Akt/mTOR, NF- $\mathrm{kB}$, Notch as well as MAPK pathways (29). Soung and Chung (29) showed that curcumin was able to inhibit cancer cell functions by disrupting the interaction between integrin $\alpha 6 \beta 4$ and EGFR. Cheng et al (12) demonstrated that curcumin could exhibit inhibitory effects on the proliferation, invasion and metastasis of prostate cancer via inhibition of MMP-9 activity, downregulation of cellular matriptase, suppression of the effects of androgens and EGFR ligands on the protease and promotion of protease shedding. Le et al (30) found that targeting EGFR with EGF-conjugated curcumin liposomes increased the antitumor activity of curcumin against pancreatic cancer cells. Treatment with curcumin effectively attenuated tobacco smoke-induced activation of the ERK and JNK MAPK pathways as well as EMT alterations in the mouse liver (31). Curcumin was also found to weaken pancreatic cancer cell growth, clonogenic potential, migration and invasion abilities by downregulation of the expression of two homologous transcriptional co-activators, YAP (Yes-associated protein) and TAZ (transcriptional coactivator with PDZ-binding motif) via the Notch signaling pathway (32). As an activator of the ligase anaphase-promoting complex/C (APC/C), cell division cycle 20 (Cdc20) plays an oncogenic role in tumorigenesis.

A recent study found that curcumin could suppress cell growth, induce apoptosis and trigger cell cycle arrest via inhibition of Cdc20 expression in pancreatic cancer cells (33). S-phase kinase associated protein 2 (Skp2) plays an oncogenic role in pancreatic cancer development and progression. Su et al (34) demonstrated that curcumin-induced cellular proliferation inhibition, cell cycle arrest, apoptosis and invasion suppression in pancreatic cancer cells was partly attributed to 
the downregulation of Skp2. Curcumin exerts its anticancer effects both alone and in combination with other anticancer drugs, including gemcitabine and 5-fluorouracil, by modulating multiple therapeutical molecular targets and signaling pathways. For instance, curcumin sensitized gemcitabineresistant cancer cells by inhibiting the expression of the PRC2 subunit EZH2 and its related lncRNA PVT1 (35).We demonstrated that curcumin suppressed hydrogen peroxideinducedcell migration and invasion through the inhibition of the ROS/ERK/NF- $\kappa \mathrm{B}$ signaling pathway (17). Curcumin was also found to inhibit superoxide dismutase-induced cancer EMT via the suppression of the PI3K/Akt/NF- $\kappa \mathrm{B}$ signaling pathway in pancreatic cancer cells (14). In the present study, our data showed that curcumin inhibited the invasive and migratory abilities of pancreatic cancer cells under high glucose conditions, which maybe attributed to the EGF/ERK and EGF/Akt signaling pathways. Our future experiments may include more in vivo experiments to further validate the findings.

In conclusion, the present study demonstrated that curcumin plays an important role in suppressing high glucose-induced proliferative, invasive and migratory abilities of pancreatic cancer cells via inhibiting the EGF/ERK and EGF/Akt signaling pathways. Curcumin might be a potential anticancer agent for the treatment of pancreatic cancer patients.

\section{Acknowledgements}

Not applicable.

\section{Funding}

The present study was supported by grants from the Natural Science Foundation of Shaanxi Province (grant serial no. 2017JM8037) and the Fundamental Research Funds for the Central Universities.

\section{Availability of data and materials}

The datasets used during the present study are available from the corresponding author upon reasonable request.

\section{Authors' contributions}

QM, ZWa and LC conceived and designed the study; WL, $\mathrm{LH}$ and $\mathrm{XX}$ conducted the experiments; and $\mathrm{ZWu}$ and $\mathrm{LC}$ performed the data analysis. WL wrote the paper. LC and QM reviewed and edited the manuscript. All authors read and approved the manuscript and agree to be accountable for all aspects of the research in ensuring that the accuracy or integrity of any part of the work are appropriately investigated and resolved.

\section{Ethics approval and consent to participate}

Not applicable.

\section{Patient consent for publication}

Not applicable.

\section{Competing interests}

The authors state that they have no competing interests.

\section{References}

1. Siegel RL, Miller KD and Jemal A: Cancer statistics, 2018. CA Cancer J Clin 68: 7-30, 2018.

2. Chen W, Zheng R, Baade PD, Zhang S, Zeng H, Bray F, Jemal A, Yu XQ and He J: Cancer statistics in China, 2015. CA Cancer J Clin 66: 115-132, 2016.

3. Michl P and Gress TM: Current concepts and novel targets in advanced pancreatic cancer. Gut 62: 317-326, 2013.

4. Bosetti C, Rosato V, Li D, Silverman D, Petersen GM, Bracci PM, Neale RE, Muscat J, Anderson K, Gallinger S, et al: Diabetes, antidiabetic medications, and pancreatic cancer risk: An analysis from the International Pancreatic Cancer Case-Control Consortium. Ann Oncol 25: 2065-2072, 2014.

5. Zhang PH, Chen ZW, Lv D, Xu YY, Gu WL, Zhang XH, Le YL, Zhu $\mathrm{HH}$ and Zhu YM: Increased risk of cancer in patients with type 2 diabetes mellitus: A retrospective cohort study in China. BMC Public Health 12: 567, 2012.

6. Lin QJ, Yang F, Jin C and Fu DL: Current status and progress of pancreatic cancer in China. World J Gastroenterol 21: 7988-8003, 2015.

7. Li J, Ma J, Han L, Xu Q, Lei J, Duan W, Li W, Wang F, Wu E, Ma Q, et al: Hyperglycemic tumor microenvironment induces perineural invasion in pancreatic cancer. Cancer Biol Ther 16: 912-921, 2015.

8. Li W,Zhang L, Chen X, Jiang Z,Zong L and Ma Q: Hyperglycemia promotes the epithelial-mesenchymal transition of pancreatic cancer via hydrogen peroxide. Oxid Med Cell Longev 2016: 5190314, 2016.

9. Appert-Collin A, Hubert P, Crémel G and Bennasroune A: Role of ErbB receptors in cancer cell migration and invasion. Front Pharmacol 6: 283, 2015.

10. Ardito CM, Grüner BM, Takeuchi KK, Lubeseder-Martellato C, Teichmann N, Mazur PK, Delgiorno KE, Carpenter ES, Halbrook CJ, Hall JC, et al: EGF receptor is required for KRASinduced pancreatic tumorigenesis. Cancer Cell 22: 304-317, 2012.

11. Han L, Ma Q, Li J, Liu H, Li W, Ma G, Xu Q, Zhou S and Wu E: High glucose promotes pancreatic cancer cell proliferation via the induction of EGF expression and transactivation of EGFR. PLoS One 6: e27074, 2011.

12. Cheng TS, Chen WC, Lin YY, Tsai CH, Liao CI, Shyu HY, Ko CJ, Tzeng SF, Huang CY, Yang PC, et al: Curcumin-targeting pericellular serine protease matriptase role in suppression of prostate cancer cell invasion, tumor growth, and metastasis. Cancer Prev Res (Phila) 6: 495-505, 2013.

13. Allegra A, Innao V, Russo S, Gerace D, Alonci A and Musolino C: Anticancer activity of curcumin and its analogues: Preclinical and clinical studies. Cancer Invest 35: 1-22, 2017.

14. Li W, Jiang Z, Xiao X, Wang Z, Wu Z, Ma Q and Cao L: Curcumin inhibits superoxide dismutase-induced epithelial-tomesenchymal transition via the PI3K/Akt/NF- $\kappa \mathrm{B}$ pathway in pancreatic cancer cells. Int J Oncol 52: 1593-1602, 2018.

15. Cao L, Xiao X, Lei J, Duan W, Ma Q and Li W: Curcumin inhibits hypoxia-induced epithelial-mesenchymal transition in pancreatic cancer cells via suppression of the hedgehog signaling pathway. Oncol Rep 35: 3728-3734, 2016.

16. Livak KJ and Schmittgen TD: Analysis of relative gene expression data using real-time quantitative PCR and the 2(-Delta Delta C(T)) Method. Methods 25: 402-408, 2001.

17. Cao L, Liu J, Zhang L, Xiao X and Li W: Curcumin inhibits $\mathrm{H}_{2} \mathrm{O}_{2}$-induced invasion and migration of human pancreatic cancer via suppression of the ERK/NF- $\kappa B$ pathway. Oncol Rep 36: 2245-2251, 2016

18. Pachmayr E, Treese $C$ and Stein U: Underlying mechanisms for distant metastasis - Molecular biology. Visc Med 33: 11-20, 2017.

19. Wu WS, Wu JR and Hu CT: Signal cross talks for sustained MAPK activation and cell migration: The potential role of reactive oxygen species. Cancer Metastasis Rev 27: 303-314, 2008.

20. Mayer IA and Arteaga CL: The PI3K/AKT pathway as a target for cancer treatment. Annu Rev Med 67: 11-28, 2016.

21. Li W, Ma Z, Ma J, Li X, Xu Q, Duan W, Chen X, Lv Y, Zhou S, Wu E, et al: Hydrogen peroxide mediates hyperglycemia-induced invasive activity via ERK and p38 MAPK in human pancreatic cancer. Oncotarget 6: 31119-31133, 2015. 
22. Jiang WG and Ablin RJ: Cancer metastasis, challenges, progress and the opportunities. Front Biosci (Elite Ed) 3: 391-394, 2011.

23. Xue C, Wyckoff J, Liang F, Sidani M, Violini S, Tsai KL, Zhang ZY, Sahai E, Condeelis J and Segall JE: Epidermal growth factor receptor overexpression results in increased tumor cell motility in vivo coordinately with enhanced intravasation and metastasis. Cancer Res 66: 192-197, 2006.

24. Cao L, Chen X, Xiao X, Ma Q and Li W: Resveratrol inhibits hyperglycemia-driven ROS-induced invasion and migration of pancreatic cancer cells via suppression of the ERK and p38 MAPK signaling pathways. Int J Oncol 49: 735-743, 2016.

25. Xu Q, Zong L, Chen X, Jiang Z, Nan L, Li J, Duan W, Lei J, Zhang L, Ma J, et al: Resveratrol in the treatment of pancreatic cancer. Ann NY Acad Sci 1348: 10-19, 2015.

26. Chiang SP, Cabrera RM and Segall JE: Tumor cell intravasation. Am J Physiol Cell Physiol 311: C1-C14, 2016.

27. Wee P and Wang Z: Epidermal growth factor receptor cell proliferation signaling pathways. Cancers (Basel) 9: 9, 2017.

28. Modjtahedi $\mathrm{H}$ and Essapen S: Epidermal growth factor receptor inhibitors in cancer treatment: Advances, challenges and opportunities. Anticancer Drugs 20: 851-855, 2009.

29. Soung $\mathrm{YH}$ and Chung J: Curcumin inhibition of the functional interaction between integrin $\alpha 6 \beta 4$ and the epidermal growth factor receptor. Mol Cancer Ther 10: 883-891, 2011.
30. Le UM, Hartman A and Pillai G: Enhanced selective cellular uptake and cytotoxicity of epidermal growth factor-conjugated liposomes containing curcumin on EGFR-overexpressed pancreatic cancer cells. J Drug Target 26: 676-683, 2018.

31. Liang Z, Wu R, Xie W, Xie C, Wu J, Geng S, Li X, Zhu M, Zhu W, Zhu J, et al: Effects of curcumin on tobacco smoke-induced hepatic MAPK pathway activation and epithelial-mesenchymal transition In Vivo. Phytother Res 31: 1230-1239, 2017.

32. Zhou X, Su J, Feng S, Wang L, Yin X, Yan J and Wang Z: Antitumor activity of curcumin is involved in down-regulation of YAP/TAZ expression in pancreatic cancer cells. Oncotarget 7: 79076-79088, 2016.

33. Zhang Y, Xue YB, Li H, Qiu D, Wang ZW and Tan SS: Inhibition of cell survival by curcumin is associated with downregulation of cell division cycle $20(\mathrm{Cdc} 20)$ in pancreatic cancer cells Nutrients 9: 9, 2017.

34. Su J, Zhou X, Wang L, Yin X and Wang Z: Curcumin inhibits cell growth and invasion and induces apoptosis through downregulation of Skp2 in pancreatic cancer cells. Am J Cancer Res 6: 1949-1962, 2016.

35. Yoshida K, Toden S, Ravindranathan P, Han H and Goel A: Curcumin sensitizes pancreatic cancer cells to gemcitabine by attenuating PRC2 subunit EZH2, and the 1ncRNA PVT1 expression. Carcinogenesis 38: 1036-1046, 2017. 